\title{
A CLASS OF STAR-SHAPED BODIES
}

\author{
Z.A. Melzak
}

(received June 26, 1959)

1. The more important properties of the class $\mathcal{K}$ of all bounded convex bodies in $\mathrm{E}_{3}$ with non-empty interior include: uniform approximability by polyhedra, existence of volume and surface area, and Blaschke's selection principle, [1], [2]. In this note we define and consider a class $\mathscr{H}$ of star-shaped bodies in $E_{3}$, which enjoys many properties of $\mathcal{K}$, among them the above-mentioned ones, and is considerably larger. Roughly speaking, $\mathcal{H}$ consists of closed bounded sets in $\mathrm{E}_{3}$ with nonempty interior, whose boundary is completely visible from every point of a set with non-empty interior. It turns out that $\mathcal{H}$ is identifiable with the class of all real-valued positive functions on the sphere $S_{3}$ which satisfy a Lipschitz condition.

Although we deal with $E_{3}$ only, extensions to $E_{n}$ and, in some respects, to infinitely dimensional spaces, present no difficulty.

Small Latin letters o, p, q, ... will denote points and capital ones will usually denote sets. Script letters will stand for classes of sets. The absolute value symbol will denote both the absolute value $|a|$ of a real number $a$ and the length $|p q|$ of the closed straight segment pq. $L(p, q)$ will be the straight line through $p$ and $q, R(p, q)$ will be the open half-line through $q$ starting at $p$, and $N(p, q)$ will be $L(p, q)-R(p, q)-p$. When $S$ is a simple closed surface we write $\mathrm{Z} \subset \mathrm{S}$ if the set $\mathrm{Z}$ is inside or on S. The empty set will be denoted by $\phi$.

2. In $E_{3}$ we consider a fixed Cartesian coordinate system with the origin o. A solid $K$ will be any bounded closed set in $E_{3}$ with non-empty interior; $B(K)$ and $I(K)$ will denote its boundary and interior respectively. Let $\sigma$ be the class of all solids $K$ such

Can. Math. Bull. vol.2, no. 3, Sept. 1959 
that $o \in I(K)$. Let $K \in \mathcal{O}, p \in K ; K$ is said to be star-shaped (or locally convex) at $p$ if $p q \subset K$ for any $q \in K$. The hub $H(K)$ of $\mathrm{K} \in \mathcal{O}$ is the set of all points at which $\mathrm{K}$ is star-shaped. The following lemma is obvious.

LEMMA 1. If $\mathrm{K} \in \boldsymbol{O}$ and $\mathrm{H}(\mathrm{K}) \neq \phi$ then $\mathrm{H}(\mathrm{K})$ is a closed convex set.

We define the class $\mathscr{H}$ as follows: $K \in \mathcal{H}$ if and only if a) $K \in O($ b) $I[H(K)] \neq \phi, c) o \in I[H(K)]$. Let $S$ be the unit sphere in $E_{3}$ about $o$ and let $K \in \mathcal{H}, s \in S$. Then $R(o s) \cap B(K)=p$; we define a function $f_{K}$ on $S$ to positive real numbers by $|o p|=f_{K}(s)$. It is clear that $f_{K}$ defines $K$ completely.

LEMMA 2. If $K \in \mathscr{H}$ then $\mathrm{f}_{\mathrm{K}}(\mathrm{s})>0$ and $\left|f_{K}\left(s_{1}\right)-f_{K}\left(s_{2}\right)\right|<\gamma_{K}\left|s_{1} s_{2}\right|, 0<\gamma_{K}<\infty$. Conversely, any such function $f$ defines a solid $K \in \mathcal{H}^{K}$.

When $Z$ is any set in $E_{3}$ and $\lambda \geqslant 0, \lambda Z$ will denote the similar and similarly located set scaled up with respect to $\circ$ in ratio $\lambda: 1$. By the hypotheses $\lambda_{1} S \subset H(K)$ and $\mathrm{K} \subset \lambda_{2} \mathrm{~S}, 0<\lambda_{1} \leqslant \lambda_{2}<\infty$. Let $\mathrm{s}_{1}$, s $2 \in \mathrm{S}$, by considering the situation in the plane os $1 s_{2}$ we see that $\left|f_{K}\left(s_{1}\right)-f_{K}\left(s_{2}\right)\right|<\lambda_{2} / \lambda_{1} \mid s_{1} s_{2} l$. If $f$ satisfies the conditions of the lemma then $f$ is continuous on $S$ and therefore uniformly continuous, and the rest follows easily.

Now we make $\mathcal{H}$ into a metric space by defining the following metric $\rho$ : for $K_{1}, K_{2} \in \mathcal{H}$ let $g(s)=f_{K_{1}}(s)-f_{K_{2}}(s)$ and put

$$
p\left(K_{1}, K_{2}\right)=\max _{s, t \in S}[|g(s)|+|g(s)-g(t)| /|s t|] .
$$

The three metric axioms are easily verified. We have

LEMMA 3. $\mathcal{H}$ is complete under $\rho$.

Let $\left\{\mathrm{K}_{\mathrm{n}}\right\}$ be a Cauchy sequence in $\mathcal{H}$. Then the set $\left\{f_{K_{n}}\right\}$ is uniformly bounded and equicontinuous on $S$. Now the lemma follows from Ascoli's Theorem and from Lemma 2.

The following theorem is an extension of Blaschke's selection principle. 
THEOREM 1. Let $\mathcal{B}$ be an infinite set in $\mathcal{H}$, such that a) if $K \in D$ then $K \subset \lambda S, 0<\lambda<\infty$, b) $I\left[\bigcap_{K \in \gg} H(K)\right] \neq \phi$. Then $\mathcal{D}$ possesses a convergent subsequence $\left\{\mathrm{K}_{\mathrm{n}}\right\}$ and $\lim \mathrm{K}_{\mathrm{n}} \in \mathcal{H}$.

Since a) and b) imply that $D$ is uniformly bounded and equicontinuous, the theorem follows from Lemma 3.

Let $S$ be the set of all polyhedra in $\mathcal{H}$. In dealing with polyhedra it will be convenient to replace the sphere $S$ by the surface $T$ of its inscribed regular tetrahedron. For $P \in P$ the function $f_{P}$ is defined on $T$, all properties relative to the Lipschitz condition hold as before, and $f_{P}$ is piecewise linear on $T$. It is simply verified that

LEMMA 4. $९$ is dense in $\mathcal{H}$.

Let $K \in \mathcal{H}$. To obtain the existence of a continuous volume $V(K)$ of $K$ and a continuous surface area $A(K)$ of $B(K)$ we take a sequence $\left\{P_{n}\right\}$ in $\mathcal{P}$, such that $\left\{P_{n}\right\} \rightarrow K$. For each $P_{n}$ the volume and surface area are defined as usual. Suppose that $P_{j}, P_{k} \subset \lambda S$, then

$$
\begin{aligned}
& \left|V\left(P_{j}\right)-V\left(P_{k}\right)\right| \leqslant(4 \pi / 3) \lambda^{3} \rho\left(P_{j}, P_{k}\right), \\
& \left|A\left(P_{j}\right)-A\left(P_{k}\right)\right| \leqslant 8 \pi \lambda^{2} \rho\left(P_{j}, P_{k}\right) .
\end{aligned}
$$

Therefore $\left\{V\left(P_{n}\right)\right\}$ and $\left\{A\left(P_{n}\right)\right\}$ are Cauchy sequences and the limits $\lim _{n} V\left(P_{n}\right)$ and $\lim _{n} A\left(P_{n}\right)$ exist and are continuous on $\mathcal{H}$.

We remark that by Lemma $4, \mathcal{H}$ is separable since the subset of $\oint$ consisting of all polyhedra all of whose vertices have all coordinates rational is countable and dense in $\mathcal{P}$.

3. In this section we consider in more detail the hub $H(K)$ of a solid $K \in \mathcal{H}$. Since $\mathrm{f}_{\mathrm{K}}(\mathrm{s})$ satisfies a Lipschitz condition on $S$, after a suitable coordinatization of $S, f(s)$ will possess partial derivatives almost everywhere. The phrase 'almost everywhere' can be used with reference to measure on $S$ or on $B(K)$ itself. In this connection see references [3] and [4]. Let $p \in B(K)$ and assume that there exists the tangent plane $\pi_{p}$ at p.

LEMMA 5. $\pi_{p}$ is bounded uniformly away from o. Since $K \in \mathcal{H}, \lambda S \subset I[H(K)], 0<\lambda$. Take $p$ and let 


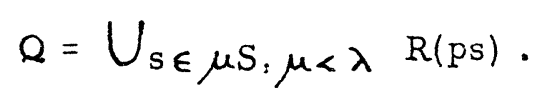

Then $\pi_{p} \cap Q=\phi, o \in Q$ and $\frac{1}{2} \lambda S \subset Q$, which proves the lemma. containing 0 .

Let $U\left(\pi_{p}\right)$ be the closed half-space bounded by $\pi_{p}$ and

$$
\text { THEOREM 2. } H(K)=\bigcap_{p} U\left(\pi_{p}\right) \text {. }
$$

The intersection is taken over all $p \in B(K)$ at which there exists a tangent plane. A similar convention applies in the sequel. Let $\mathrm{q} \in I[\mathrm{H}(\mathrm{K})]$; as in Lemma 5 it can be shown that there exists a sphere $V$ about $q$ disjoint from every $\pi_{p}$. It follows that $q \in \cap_{p} U\left(\pi_{p}\right)$, therefore $I[H(K)] \subset \cap_{p} U\left(\pi_{p}\right)$, and since the intersection of closed sets is closed, $H(K) \subset \bigcap_{p} U\left(\pi_{p}\right)$.

Now let $q \in I\left(\cap_{p} U\left(\pi_{p}\right)\right)$ and take $t \in K$. Suppose that $\not K$. The set $X=\{x \mid x \in q t, x \notin K\}$ is open and therefore ost countable union of intervals. Let $(u, v)$ be one such Lt the vagent planes $\pi_{u}$ and $\pi_{V}$ exist then $q \notin-\left[\mathrm{U}\left(\pi_{\mathrm{u}}\right) \cap \mathrm{U}\left(\pi_{\mathrm{v}}\right)\right]$ which is a contradiction. But even if $\pi_{u}$ and $\pi_{v}$ do not exist, one can always select points $u_{1}$ and $v_{1}$, at which tangent planes exist, and which are arbitrarily close to $u$ and $v$ respectively. Now the contradiction is estab. lished as before.

Therefore qt $\in K$ for every $t \in K$. It follows in succes sion that $q \in H(K), I\left(\cap_{p} U\left(\pi_{p}\right)\right)<H(K), \cap_{p} U\left(\pi_{p}\right) \subset H(K)$, and so $\bigcap_{\mathrm{P}} \mathrm{U}\left(\pi_{\mathrm{p}}\right)=\mathrm{H}(\mathrm{K})$. Il to $\mathscr{H}$.

COROLLARY. The function $\mathrm{K} \rightarrow \mathrm{H}(\mathrm{K})$ is continuous on

Theorem 2 shows that the concept of hub of a set is in a certain sense dual to that of convex hull.

THEOREM 3. Given any convex $C \in \mathcal{H}$, any $\lambda>0$ such that $C<\lambda S$, and any $\varepsilon>0$, there exists $K \in X$ such that a) $H(K)=C$, b) $C \subset I(K)$, c) $\lambda S \in K \in(\lambda+\varepsilon) S$.

Let $\left\{s_{n}\right\}, n=1,2, \ldots$ be a denee sequence of points on S. Let $\left\{\lambda_{n}\right\}, n=1,2, \ldots$ be a monotone increasing sequence of real numbers, such that $\lambda<\lambda_{1}$ and $\lim \lambda_{n}=\lambda+\varepsilon$. 
Let $p_{n}=R\left(\operatorname{os}_{n}\right) \cap \lambda_{n} S$ and let

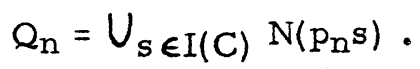

Let $(\lambda+\varepsilon) S=B(Z), \quad Z \in \mathcal{H}$, and put

$$
\mathrm{K}=\mathrm{Z}-\mathrm{U}_{\mathrm{n}}^{\infty} \stackrel{\infty}{=} \mathrm{Q}_{\mathrm{n}} \text {. }
$$

The above-defined solid $K$ satisfies the conditions of the theorem. This construction could be varied in many ways and the theorem could be exterided. For example, one could take in c) any $B\left(K_{1}\right)$ instead of $S$, subject to the condition $\mathrm{C} \subset \mathrm{H}\left(\mathrm{K}_{1}\right)$.

Theorem 3 suggests that if $H(K) \subset I(K)$ and $B(K)$ is a fairly smooth surface, then $H(K)$ ought to be a fairly special type of a convex set (this is taken with particular reference to the set of its extreme points).

Let $\mathrm{K} \in \mathcal{H}$, a set $\mathrm{Y} \subset \mathrm{B}(\mathrm{K})$ is called enveloping if $H(K)=\bigcap_{p \in Y} U\left(\pi_{p}\right)$, and it is called essential if it is enveloping, closed and possesses no proper enveloping subset. Since the enveloping sets of $B(K)$ are partially ordered by inclusion and $B(K)$ is one of them, the existence of at least one essential set follows from Zorn's Lemma. It is clear that if a tangent plane exists at all $p \in B(K)$ and $Y$ is enveloping, then $Y$ is essential only if $\pi_{p} \cap \mathrm{H}(\mathrm{K}) \neq \phi$ for every $\mathrm{p} \in \mathrm{Y}$. Further, if $B(K)$ is essential the $K$ is convex and every point $p \in B(K)$ is extreme.

A simple closed surface $W$ in $E_{3}$ will be called smooth if it is of class $C^{2}$ and if the sets $W_{E}$ and $W_{H}$ of its elliptic and its hyperbolic points are both open. $W_{P}$ will denote the set of parabolic points of $\mathrm{W}$.

THEOREM 4. Let $K \in \mathcal{H}, H(K) \subset I(K)$ and suppose that $B=B(K)$ is smooth. If $Y \subset B$ is essential then $Y \subset B P$.

Suppose that $p \in Y \cap B_{E}$. Assume first that the vector normal to $B$ at $p$ points outward. If $\pi_{p} \cap B=p$ then it follows that $p \in H(K)$, which is a contradiction since $H(K) \subset I(K)$. If $\pi p \cap B$ contains a point $q \neq p$, then there exists a point $t \in B$, such that $\pi_{t}$ is parallel to $\pi_{p}$ and closer to o than $\pi_{p}$. This contradicts the assumption $p \in Y$.

Assume now that the vector normal to $B$ at $p$ points inward. Let $C$ be a small simple closed curve on $B$ encircling $p$, for 
instance, a geodesic circle of small radius about $p$. Let $V=\bigcap_{c \in C} U\left(\pi_{c}\right)$, it is easy to verify that $V$ is disjoint from $\pi_{p}$. By Theorem $2 \mathrm{H}(\mathrm{K}) \subset \mathrm{V}$, therefore $\pi_{\mathrm{p}}$ is disjoint from $H(\mathrm{~K})$ which again contradicts the assumption $\mathrm{p} \in \mathrm{Y}$.

It can be proved by almost exactly the same means that if $p \in B_{H}$ then $p \notin Y$. Since $B=B_{E} \cup B_{H} \cup B_{P}$ and the three summands are disjoint, the proof is complete.

COROLLARY. If $B_{p}$ consists of a finite number of arcs, not necessarily disjoint, then $\mathrm{H}(\mathrm{K})$ is bounded by a finite number of developable surfaces. Therefore the extreme points of $H(K)$, like the parabolic points of $\mathrm{K}$, lie on a finite number of arcs.

Let $C$ be a subarc of $B_{p}$. Then the tangents to $B$ along $C$ envelop a surface which is a one-parameter family of straight lines. It follows that $\mathrm{B}[\mathrm{H}(\mathrm{K})]$ is bounded by a finite number of ruled surfaces. However, $\mathrm{H}(\mathrm{K})$ is convex and a convex ruled surface is developable. The rest follows from the definitions of an extreme point and a developable surface.

\section{REFERENCES}

1. T. Bonnesen and W. Fenchel, Konvexe Koerper, (Chelsea, 1948).

2. W. Blaschke, Kreis und Kugel, (Chelsea, 1949).

3. H. Rademacher, Partielle and totale Differenzierbarkeit, Math. Annalen 81 (1920), 52 .

4. T. Rado, Flaechenmass rectifizierbarer Flaechen, Math. Annalen 100 (1928), 445.

McGill University 